\title{
Body-specificity Effects on Different Bodily Traits
}

\author{
Chong $\mathrm{Liu}^{1, *}$, Yueyue $\mathrm{Li}^{2}$, Zhizhuo $\mathrm{Wu}^{3}$ \\ ${ }^{1}$ School of Education, Nanjing University, Nanjing 210023, China \\ ${ }^{2}$ School of Education, University of California, Irvine, Irvine 92614, United States of America, yueyul2@uci.edu \\ ${ }^{3}$ WLSA Shanghai Academy, Shanghai 200243,China,tfvvft@163.com \\ *Corresponding author. Email: chong.liu@smail.nju.edu.cn
}

\begin{abstract}
According to the body-specificity hypothesis, people with different kinds of bodies should think differently. Since this hypothesis was proposed, a growing body of experimental evidences support it. However, the types of physical or empirical characteristics involved at present are relatively limited, and handedness is the main one, while other bodily traits are seldom studied. In addition, most of the current studies have only found the correlation between physical characteristics and cognition, while the causal relationship between them has not been extensively and deeply studied. This article reviews the major studies that have shown body-specificity effects on different bodily traits and make some recommendation for further research.
\end{abstract}

Keywords: body-specificity hypothesis, bodily traits, handedness, footedness, overweight, blindness.

\section{INTRODUCTION}

Do differences in physical characteristics lead to different cognitive patterns by leading to systematic differences in how people interact with the physical environment? Casasanto [1] thought the answer may be yes, and called this proposal the body-specificity hypothesis. This hypothesis provides us a tool to find out how knowledge might be dependent upon our interactions with the environment. Since the bodyspecificity hypothesis was proposed, more and more studies have tested it. This paper reviews these studies in terms of different physical characteristics and makes some recommendations for further research.

\section{BODY-SPECIFICITY EFFECTS ON DIFFERENT BODILY TRAITS}

\section{1 handedness}

Most of the current studies have examined the relationship between handedness and cognitive differences. Some studies have shown that right-handed people tend to associate "right" with positive thoughts and "left" with negative thoughts, while left-handed people show the opposite pattern, preferring to associate "left" with positive thoughts and "right" with negative thoughts [2-5], and even children as young as five show similar patterns [6]. This association between valence and left/right is potentially biased in political elections, in which people are more likely to vote for candidates whose names are closer to their dominant side [7]. The fluency of the movement is considered to be the key factor affecting the mapping. Perhaps people tend to associate positive thoughts with the dominant side of a space because they can interact with the environment more easily with their dominant hand, and people like objects that are easier to move [8]. Therefore, the fluency of movement is considered to be the key factor affecting the mapping, which has been confirmed by experiments. Some experiments have shown that this mapping is malleable and can be reversed in the short term by participants' imagination or by interventions for motor fluency, suggesting that physical features and physical experiences can trigger or shape cognitive features $[9,10]$.

\section{2 footedness}

Similar to people with dominant hand, people will also perform tasks differently with their left or right dominant foot. So, are different foot preferences in our populations a factor for the way people think and associate? de la Vega et al. [11] tested this by recording precise reaction times of people when responding to positive and negative word stimulus with their feet. In the experiments, all participants were right-handers and right-footers. The participants were asked to make a valence judgment to words and use their right foot for positive valence and left for negative for the first period and reversed for the second period. The result confirmed 
the hypothesis by showing that right footers respond faster to positive valence words with their right foot, and negative with their left foot. Further analyzing the results, the experimenters found out that the association between valence and left, right foot is only significant in strong right footers, but not in weak right-footers. This experiment had verified the footedness effect of right footers, suggesting that the strength of right footedness tendency has a positive correlation towards valence judgment. However, two possibilities that this result does not explain are the influence of the overlapping right handedness on right footed people and the effect of left footedness on people's valence judgement. the result of this experiment might not rule out a competitive explanation: the handedness of those participants would lead them to a non-foot related valence judgement. Even when the participants were asked to use foot pedals, controlled only by their foot motions, the valence judgement can be an effect of handedness instead of footedness. Partially, the further analysis of the result gives a credible clarification to reject this altered explanation. If the result is caused by the participants' handedness, then the strength of right footedness would not be a variable influencing the result, but significant difference is observed that valence association is only present in strong right footers, but not in weak ones. Furthermore, as Elias et al. [12] showed, footedness is confirmed to be a better predictor than handedness of emotional lateralization, and for cases where its participants were cross lateralized, the only result that is significant for footedness. Both of them suggested that footedness is qualified to be a variable independent from the overlapping of handedness and can be responsible for valence judgement, refuting the hypothesis that handedness is the actual factor of influence for the results.

\section{3 overweight}

While handedness and footedness, which are mainly in control of our motor actions, shape our perceptions, can bodily traits that are not motor related such as weight also play a role in influencing our representation and reaction of environmental stimuli? Some studies showed that there is supposedly a stronger connection between being overweight and biases towards food stimuli in overweight children's populations [13, 14], but other studies failed to find a similar relationship [15]. Tárrega et al. [16] adopted a new lexical decision task in place of bias assessing test, which means the participants would have to identify whether the word they perceived is a word or not. They observed the pattern that overweight children reacting faster to food related words and this pattern is negative in children with healthy weight. Although this pattern is observed among overweight children, there are no previous studies addressing the same problem for adults using a similar method as this study. Those that had addressed it using other methods including all of the bias assessing test have failed to prove a positive correlation for adults. Therefore, even if it is plausible to conclude that weight as a bodily trait can influence children's perception and reaction, the result cannot be generalized to the adult population before further in-depth researches attain a more thorough understanding on this topic.

\section{4 blindness}

Bottini et al. [17] tested the differences in the cognitive representation of sequences in working memory among people with normal vision, early blindness, and late blindness. First, all participants were blindfolded and asked to listen to a series of words (either fruit or vegetable) and memorize them in the correct order. New words were then mixed into the series, and all the words were played in a random order. The participants were asked to decide whether each word had been heard in the previous stage, and if they thought they had, they needed to classify the word as a fruit or vegetable by pressing a button on the left or right. The results showed that items at the beginning of the sequence were classified faster using the left button, and items at the end of the sequence were classified faster using the right button, both for sighted and late blind participants (blindness in adults or after age 3, with visual memory). In contrast, early blind participants (those who lose their sight at birth or before age 3, have no visual memory and have never used their vision functionally) did not show this pattern. This suggests that differences in visual ability affect people's cognitive characteristics in dealing with ordered items, and early blindness can alter the spatial organization of verbal working memory.

Iversen et al. [18] compared the olfactory perception of people who were blind from birth with that of sighted people. The results showed that people who were blind from birth were more likely to identify fear and disgust based on the smell of a man's sweat than those who were sighted. In addition, a number of studies have shown that blind people seem to be more sensitive to non-visual information than sighted people [19]. These can all be seen as evidence that differences in visual ability lead to cognitive differences.

\section{5 specific experience}

Based on the body-specificity hypothesis, the reason why specific physical characteristics can lead to specific cognitive characteristics is that they first lead to specific bodily experience and then leads to specific cognitive patterns through direct bodily experience. There are some empirical studies that examine the effects of specific bodily experience on cognition, although they do not directly examine bodily traits. We think that they can also be seen as evidences that support an important part of overall body-specificity hypothesis. 
For example, only pianists have extensive and specific sensorimotor experiences with piano keyboards, and this specific physical experience may cause them to have different cognitive representation patterns of pitch than non-pianists. An experimental study tested this [20]. First, participants were shown sentences associated with high or low pitches on a computer screen, either describing high-pitched events (e.g. The monkeys screech shrilly), low-pitched events (e.g. The turbines are emitting a hollow drone), or nonsense sentences (e.g. The clarinet plays a low pot). Then, they were instructed to read the sentences, and judge, as quickly as possible, whether the sentence made sense, and enter the answer by pressing a button on the left or right (in some groups, right button meant "this sentence is sensible"; in others, it meant "this sentence is nonsense"). For example, if a participant read a sentence and judged that it was describing a high-pitched event, and the right button in this group meant sensible, he should press the right button rather than the left one. The results showed that pianists responded faster to sentences describing low-pitched auditory events when they used the left key than when they used the right key. In contrast, they responded faster to sentences describing high-pitched auditory events when they responded with the right button than when they responded with the left button. The non-musicians showed no difference in how fast they responded to similar situations. This result suggests that when understanding concepts related to pitch, pianists mentally associate pitch with left and right Spaces, whereas nonpianists do not, most likely due to the production of highpitched sounds on the right side of the piano keyboard and low-pitched sounds on the left.

\section{DISCUSSION}

It is necessary to realize that, first of all, the physical characteristics examined so far are relatively limited, and handedness is the main one, while other bodily traits are seldom studied. Secondly, most of the current researches get correlation rather than causality. Except when handedness is taken as the experimental platform, a few researches confirm that the difference in handedness in some situations is the cause of cognitive specificity through true experiments [9]. Most other studies have only revealed correlations between differences in physical characteristics and differences in cognition, rather than causation. Therefore, the relevant validation needs to be further expanded and deepened.

Regarding further testbeds, we have conceived two dimensions. As shown in Table 1, the first dimension is about bodily traits, such as vision, hearing, height, weight, etc.; the second dimension involves cognitive characteristics, such as sense of time, sense of space, sense of orientation, sense of value, intensity of attention, sensitivity of perception, degree of trust, etc. Different parts of these two dimensions can be combined arbitrarily to form a possible relationship between bodily traits and cognitive specificity, which will lead to further experimental verification. For example, here are some possible hypotheses: Blindness may cause changes in attention patterns; Blindness may lead to differing confidence in evidence (after hearing the same testimony); Height may affect a person's self-confidence, so students' awe of their teachers may be related to their height; People with different weights will have different judgments about how dangerous the same thing is.

\section{Table 1. Further testbeds for body-specificity effects}

\begin{tabular}{l|l}
\hline \multicolumn{1}{c}{ Bodily traits } & \multicolumn{1}{c}{ Consequences for minds } \\
\hline vision: red-green color blindness, \\
early/late blindness, normal vision \\
hearing: deafness, normal hearing \\
height: tall, short
\end{tabular}

As for the way of research, the existing research methods can be used for reference, and at least two ways can be roughly carried out. The first is correlational studies, which investigate the correlation between physical specificity and cognitive specificity. The second approach is to explore causality, that is, through true experiments, to study whether specific physical characteristics are functional causes that regulate or trigger specific cognitive patterns. In the second path, the experimental paradigm that can be referred to is from Fuente et al. [9] in which the researchers changed the mobility of the left and right hands by putting clumsy gloves on the participants, and then tested whether this physical experience produced observable differences in cognitive outcomes. Similarly, in the study of other physical characteristics, we suggest that researchers can 
change the action ability of the corresponding physical characteristics through experimental conditions and carry out true experiments with control variables. For example, to test the effect of visual features on cognitive features, visually normal people could wear blindfolds to create a short-term experience of visual blindness, and then test their cognitive predispositions to certain activities. For another example, if the effect of body shape or height on cognitive features is verified, the body shape or height perceived by the subject can be changed by making the participants wear equipment to make their bodies appear larger, or by wearing virtual reality devices.

\section{CONCLUSION}

In summary, a growing body of experimental evidences support the main idea of the body-specificity hypothesis: that different physical characteristics lead to different cognitive patterns (at least in some cognitive activities, if more rigorously stated). However, the types of physical or empirical characteristics involved at present are relatively limited, and this hypothesis needs to be explored in a wider range in the future. In addition, most of the current researches only get the correlation between physical characteristics and cognition. In order to understand the impact of physical or empirical characteristics on cognition, more true experiments are needed to explore the causal relationship.

Although the body-specificity hypothesis was originally proposed based on embodied cognitive theory [1], we believe that this topic can transcend the debate between embodied and disembodied theories, because it seems that many of the results can be explained by both the embodied theories and the amodal alternatives. Perhaps the greatest value of this type of research lies in examining biases and limitations in human cognition, particularly the relationship between people's propensity to evaluate and the specificity of their body or experience.

\section{AUTHOR CONTRIBUTIONS}

Chong Liu planned the structure of the paper, participated in literature collection, wrote the first draft of section 2.4, 2.5, 3 and 4, provided guidance and suggestions for the first draft of other parts, substantially rewrote section 1 and 2.1, and consolidated and revised the full text. Yueyue $\mathrm{Li}$ participated in literature collection, wrote the first draft of section 1 and 2.1, and revised section 2.2 and 2.3. Zhizhuo $\mathrm{Wu}$ participated in the literature collection, and wrote and revised section 2.2 and 2.3 .

\section{REFERENCES}

[1] Casasanto, D. (2009). Embodiment of abstract concepts: good and bad in right- and left-handers. Journal of experimental psychology. General. https://doi.org/10.1037/a0015854
[2] Casasanto, D. (2011). Different Bodies, Different Minds: The Body Specificity of Language and Thought. Current Directions in Psychological Science, 20(6), 378-383. https://doi.org/10.1177/0963721411422058

[3] Casasanto, D., \& Chrysikou, E. G. (2011). When Left Is "Right": Motor Fluency Shapes Abstract Concepts. Psychological Science, 22(4), 419-422. https://doi.org/10.1177/0956797611401755

[4] Casasanto, D., \& Jasmin, K. (2010). Good and Bad in the Hands of Politicians: Spontaneous Gestures during Positive and Negative Speech. PLOS ONE, $5(7)$,

e11805. https://doi.org/10.1371/journal.pone.0011805

[5] Loffing, F., Prelle, L., Heil, L., \& Cañal-Bruland, R. (2019). Body-specific influences on performance evaluation in realistic dynamic scenes. Laterality. https://doi.org/10.1080/1357650X.2018.1522323

[6] Casasanto, D., \& Henetz, T. (2012). Handedness Shapes Children's Abstract Concepts. Cognitive Science, 36(2), 359-372. https://doi.org/10.1111/j.1551-6709.2011.01199.x

[7] Kim, N., Krosnick, J., \& Casasanto, D. (2015). Moderators of Candidate Name-Order Effects in Elections: An Experiment: Moderators of Candidate Name-Order Effects. Political Psychology, 36(5), 525-542. https://doi.org/10.1111/pops.12178

[8] Ping, R. M., Dhillon, S., \& Beilock, S. L. (2009). Reach For What You Like: The Body's Role in Shaping Preferences. Emotion Review, 1(2), 140 150. https://doi.org/10.1177/1754073908100439

[9] Fuente, J. de la, Casasanto, D., \& Santiago, J. (2015). Observed actions affect body-specific associations between space and valence. Acta psychologica. https://doi.org/10.1016/j.actpsy.2015.01.004

[10] Fuente, J., Casasanto, D., Martínez-Cascales, J. I., \& Santiago, J. (2017). Motor Imagery Shapes Abstract Concepts. Cognitive Science, 41(5), 1350 1360. https://doi.org/10.1111/cogs.12406

[11] de la Vega, I., Graebe, J., HÃ.ortner, L., Dudschig, C., \& Kaup, B. (2015). Starting off on the right foot: strong right-footers respond faster with the right foot to positive words and with the left foot to negative words. Frontiers in Psychology, 6 . https://doi.org/10.3389/fpsyg.2015.00292

[12] Elias, L. J., Bryden, M. P., \& Bulman-Fleming, M. B. (1998). Footedness is a better predictor than is handedness of emotional lateralization. Neuropsychologia, 36(1), 37-43. https://doi.org/10.1016/s0028-3932(97)00107-3 
[13] Koch, A., \& Pollatos, O. (2014). Interoceptive sensitivity, body weight and eating behavior in children: a prospective study. Frontiers in Psychology, https://doi.org/10.3389/fpsyg.2014.01003

[14] Werthmann, J., Jansen, A., \& Roefs, A. (2015). Worry or craving? A selective review of evidence for food-related attention biases in obese individuals, eating-disorder patients, restrained eaters and healthy samples. PROCEEDINGS OF THE NUTRITION SOCIETY, 74(2). https://doi.org/10.1017/s0029665114001451

[15] Soetens, B., \& Braet, C. (2007). Information processing of food cues in overweight and normal weight adolescents. British Journal of Health Psychology, 12(Pt 2), 285-304. https://doi.org/10.1348/135910706X107604

[16] Tárrega, J., Perea, M., Rojo-Bofill, L. M., MorenoGiménez, A., Almansa-Tomás, B., Vento, M., \& García-Blanco, A. (2021). Do children with overweight respond faster to food-related words? Appetite, 161, 105134. https://doi.org/10.1016/j.appet.2021.105134

[17] Bottini, R., Mattioni, S., \& Collignon, O. (2016). Early blindness alters the spatial organization of verbal working memory. Cortex. https://doi.org/10.1016/j.cortex.2016.08.007

[18] Iversen, K. D., Ptito, M., Møller, P., \& Kupers, R. (2015). Enhanced Chemosensory Detection of Negative Emotions in Congenital Blindness. Neural Plasticity, 2015, 1-7. https://doi.org/10.1155/2015/469750

[19] Crollen, V., \& Collignon, O. (2012). Embodied Space in Early Blind Individuals. Frontiers in Psychology, https://doi.org/10.3389/fpsyg.2012.00272

[20] Wolter, S., Dudschig, C., \& Kaup, B. (2016). Reading sentences describing high- or low-pitched auditory events: only pianists show evidence for a horizontal space-pitch association. Psychological Research. $\quad$ https://doi.org/10.1007/s00426-0160812-z 